\title{
ASSESSMENT OF SEXUAL ACTIVITY LEVELS AND THEIR ASSOCIATION WITH EJACULATE PARAMETERS IN TWO-BREED HYBRIDS AND PUREBRED DUROC AND PIETRAIN BOARS*
}

\author{
Anna Wysokińska+, Stanisław Kondracki \\ Department of Animal Reproduction and Hygiene, \\ Siedlce University of Natural Sciences and Humanities, Prusa 14, 08-110 Siedlce, Poland \\ •Corresponding author: annaw@uph.edu.pl
}

\begin{abstract}
The aim of the present study was to analyse sexual activity levels of two purebred boars (Pietrain and Duroc) in comparison with two-breed hybrid boars (Duroc $\odot \times$ Pietrain $\delta$ and Pietrain $\odot \times$ $\times$ Duroc ${ }^{-}$) and to assess the relationship between the ejaculate parameters and the sexual activity of the animals. The experimental material was constituted by 20 boars. Sexual activity measurements were performed in $\mathbf{4}$ different collection periods for each boar. The first libido analysis was carried out during the first week of insemination use, followed by the next analyses after 3, 6 and 9 months of service. In parallel to the sexual activity analyses, ejaculates were collected to investigate ejaculatory efficiency, physical characteristics of the ejaculates and sperm morphology. It has been shown that the characteristics of sexual activity parameters are affected by the breed of the boar and the crossbreeding variant. Two-breed sires are quicker to leap onto the phantom as compared with purebred boars. Duroc $q \times$ Pietrain ${ }^{\lambda}$ boars are characterized by a short period of time between phantom mounting and ejaculation initiation. On the other hand, they yield ejaculates for a much longer time than hybrid Pietrain $q \times$ Duroc $^{\dagger}$ boars and purebred Duroc and Pietrain boars. Hybrid boar ejaculates contain more spermatozoa and can provide more insemination doses than ejaculates of purebred boars. Ejaculate parameters are chiefly correlated with ejaculation time and copulation duration. In our study the ejaculate parameters (ejaculate volume, sperm concentration and sperm motility) in purebred boars mainly depended on ejaculation time and total copulation duration, whereas in two-breed hybrids they were more affected by the length of the period between phantom mounting and the start of ejaculation.
\end{abstract}

Key words: pigs, boars, libido, semen

Artificial insemination is an efficacious breeding method that facilitates fast genetic improvement of pig populations. Genetic progress is far more conditioned by

*This study was financed from statutory activity. 
males than females. The following reproduction performance traits of boars are practically important for artificial insemination: sexual activity, and the volume and quality of produced semen. These traits exhibit high variability and significantly affect the performance value of insemination boars (Chamberlain and Hughes, 1996; Levis et al., 1997). The release speed and course of copulatory reflexes is conditioned by genetic factors. However, there are numerous environmental factors that affect sexual activity as well (Petherick, 2005; Galina et al., 2007). The environmental factors influencing reproduction include seasonal factors like ambient temperature and day length (Ciereszko et al., 2000; Knecht et al., 2013). The sexual behaviour of males depends on the complex interaction between the organism and the environment. The understanding of sexual behaviour regulation is important for the optimisation of boar reproductive efficiency (Hemsworth and Tilbrook, 2007). Genetic factors that affect both semen parameters and libido include sire breed. Numerous studies have shown that representatives of particular breeds produce ejaculates that differ qualitatively and quantitatively (Ciereszko et al., 2000; Wolf and Smital, 2009; Kondracki et al., 2012). It has also been ascertained that hybrid boars display pronounced sexual urge and easily produce ejaculates, which is essential for insemination practice. They are also generally in a good shape, with higher resistance in comparison with purebred boars (Sonderman and Luebbe, 2008). It has also been observed that ejaculates obtained from hybrids have better both quantitative and qualitative parameters as compared with ejaculates of purebred boars (Smital et al., 2004; Kawęcka et al., 2008; Wysokińska et al., 2009). The sperm used in insemination procedures very often comes from the crossbred boars. There is a constant and substantial demand on their semen. The purchase of insemination boars requires a considerable financial outlay. That is why it is extremely important that their performance value is adequate. It is quite probable that libido measurements can be used for the assessment of boar insemination usefulness, with conclusions concerning ejaculate quality drawn on the basis of the relationship between ejaculate parameters and sexual activity. Unfortunately there are not many studies of libido levels and libido relationship with ejaculate parameters available, while their results are far from conclusive.

The present study was aimed at analysing sexual activity levels of two purebred boars (Pietrain and Duroc) in comparison with two-breed hybrid boars (Duroc $q \times$ $\times$ Pietrain $\delta^{\lambda}$ and Pietrain $+\times \operatorname{Duroc}^{\widehat{ }}{ }^{\lambda}$ ) and assessing the relationship between the ejaculate parameters and the sexual activity of the animals.

\section{Material and methods}

The experimental material was constituted by 20 insemination boars, including 5 Duroc and Pietrain males and 5 Duroc $q \times \operatorname{Pietrain}^{\lambda}$ and Pietrain $q \times \operatorname{Duroc} \hat{\sigma}$ hybrids, respectively used in three sow artificial insemination centres owned by the Mazovian Centre of Animal Breeding and Reproduction in Lowicz (Poland). All the boars included in the studies were maintained in standard-conditioned sow artificial 
insemination centres, where they were analogically fed and nurtured. The sperm was collected in accordance with the procedure uniformly applied in the sow artificial insemination centre. The study involved young boars aged 7-8 months that were on the threshold of their reproduction service. Sexual activity measurements were performed in 4 different collection periods for each boar. The first libido analysis was carried out during the first week of insemination service, followed by the next analyses after 3, 6 and 9 months of service. The measurements were conducted in the morning between 6:30 and 8:30. The sexual activity of the boars was assessed based on the time when the successive copulatory reflexes were released during manual semen collection. The following sexual activity parameters were selected for the assessment: time from the boar entering the arena until mounting the phantom, time from phantom mounting until the start of ejaculation, duration of ejaculation, total copulation time, i.e. the time from effectively mounting the phantom until dismounting following completed ejaculation, number of climbs onto the phantom necessary to yield semen. The duration of sexual reflexes was determined using a stop-watch exact to one second.

In parallel to the sexual activity analyses, ejaculates were collected to investigate ejaculatory efficiency, physical characteristics of the ejaculates and sperm morphology. The ejaculates were manually collected into germ-free plastic bags placed in heat-insulating containers sealed with disposable viscose filters. This made it possible to directly separate the gelatinous fraction. Immediately after collection, the ejaculates were analysed for the following physical parameters: ejaculate volume, sperm concentration, percentage of spermatozoa with correct motility, total number of spermatozoa per ejaculate and the number of insemination doses obtained from a single ejaculate. Ejaculate volumes were determined after filtrating the gelatinous fraction off, using electronic scales to measure ejaculate weight. Sperm concentration in the ejaculates was determined with a photometric method, using a spectrophotometer (IMV Technologies, France). The percentage of correctly motile spermatozoa was identified microscopically. A drop of fresh semen was placed on a microscope slide heated to $37^{\circ} \mathrm{C}$. A light microscope with a heated stage was used for the analyses. At a 200-fold zoom, the percentage share of correctly motile spermatozoa was determined in the overall number of spermatozoa in the field of vision. The total number of motile spermatozoa in the ejaculates and the number of insemination doses obtained from a single ejaculate were calculated using SYSTEM SUL (v. 6.35; Gogosystem, Poland) computer program. Moreover, the samples of each ejaculate were used to make microscopic preparations in which the morphological structure of 500 spermatozoa was assessed. The number of well-formed and morphologically aberrant spermatozoa was established, with further specification of spermatozoa exhibiting primary and secondary alterations, following Blom's classification (Blom, 1981).

Experimental data were analysed using STATISTICA 10 PL program (StatSoft, Tulsa, USA). The material obtained was statistically analysed according to the following mathematical model: 


$$
Y_{i j k}=\mu+a_{i}+b_{j}+e_{i j k}
$$

where:

$Y_{i j k}$ - value of the parameter under analysis,

$\mu$ - overall mean,

$a_{i}$ - boar breed effect,

$b_{j}-$ collection period effect,

$e_{i j k}-$ random error.

The significance of the differences between the groups was assessed with the Tukey's test at $\mathrm{P} \leq 0.05$. Additionally, phenotypic correlation coefficients were calculated on the basis of Pearson linear correlation between the sexual activity parameters and physical ejaculate parameters and the incidence of morphological changes in the spermatozoa.

\section{Results}

Table 1 shows the results of the analyses of sexual activity and the evaluation of the ejaculates and sperm morphology. The hybrids needed less time between entering the arena and effectively mounting the phantom than the purebred boars. The Duroc boars had the shortest times between mounting the phantom and initiating ejaculation, as well as much shorter ejaculation and copulation times, with a slightly higher number of leaps onto the phantom necessary to produce semen as compared with the Pietrain boars and the two-breed hybrids. The hybrid boars Duroc $q \times$ Pietrain $\delta$ needed a relatively short period between mounting the phantom and starting ejaculation ( 85.30 seconds) and, on the other hand, much more time for ejaculation in comparison with the purebred Duroc and Pietrain boars, as well as the Pietrain 9 $\times$ Duroc $\delta$ hybrids. Both types of hybrids and the Pietrain sires were found to have significantly longer copulation times than the Duroc boars $(\mathrm{P} \leq 0.05)$. The Duroc $q$ $\times$ Pietrain $\widehat{\gamma}$ crosses produced ejaculates with a volume similar to the volume of the Pietrain boar ejaculates. On the other hand, the Pietrain $\uparrow \times$ Duroc $\widehat{\sigma}$ crosses provided ejaculates with a volume similar to the volume of the Duroc boar ejaculates. This suggests that ejaculate volume of hybrid boars is conditioned to a greater degree by the paternal rather than the maternal breed. Pietrain $q \times$ Duroc $\widehat{\gamma}$ boars produced ejaculates with lower volumes (by $39.40 \mathrm{ml}$ ) than those of the Duroc $q \times$ $\times$ Pietrain ${ }^{\lambda}$. The hybrid boar ejaculates had high sperm concentrations, similar to the concentration of spermatozoa in the ejaculates of the Duroc boars and much higher than the concentration of spermatozoa in the Pietrain ejaculates. They also had a high ejaculate sperm count, by $4.31-15.65 \times 10^{9}$ higher than the purebred ejaculates, and produced a higher number of insemination doses from a single ejaculate. Duroc semen was observed to contain the highest number of spermatozoa with secondary morphological alterations. The hybrids produced semen with a small percentage of morphological aberrations. The percentage of well-formed spermatozoa in hybrid semen was very high, slightly higher than in the semen of the Pietrain boars and approximately $6-8 \%$ higher than in the semen of the Duroc boars $(\mathrm{P} \leq 0.05)$. 


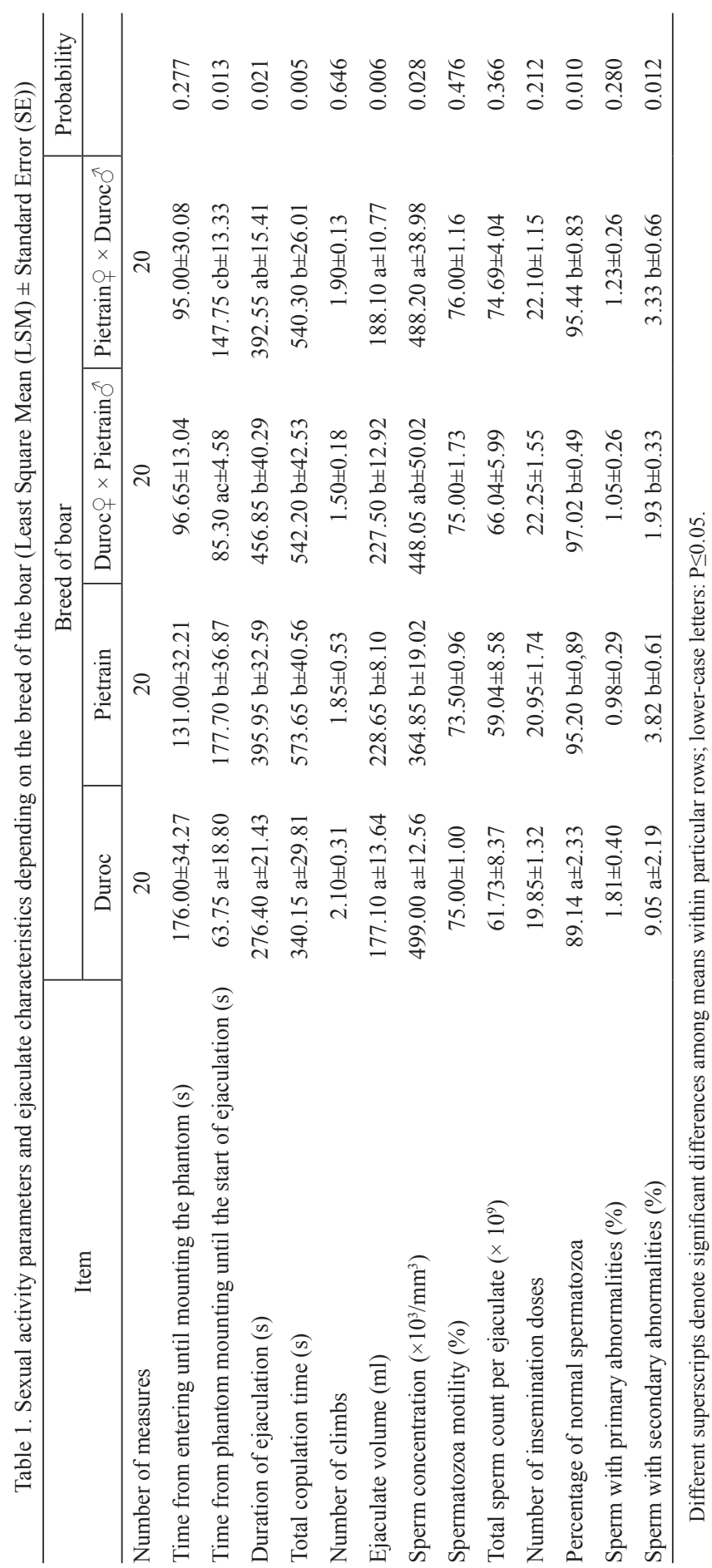




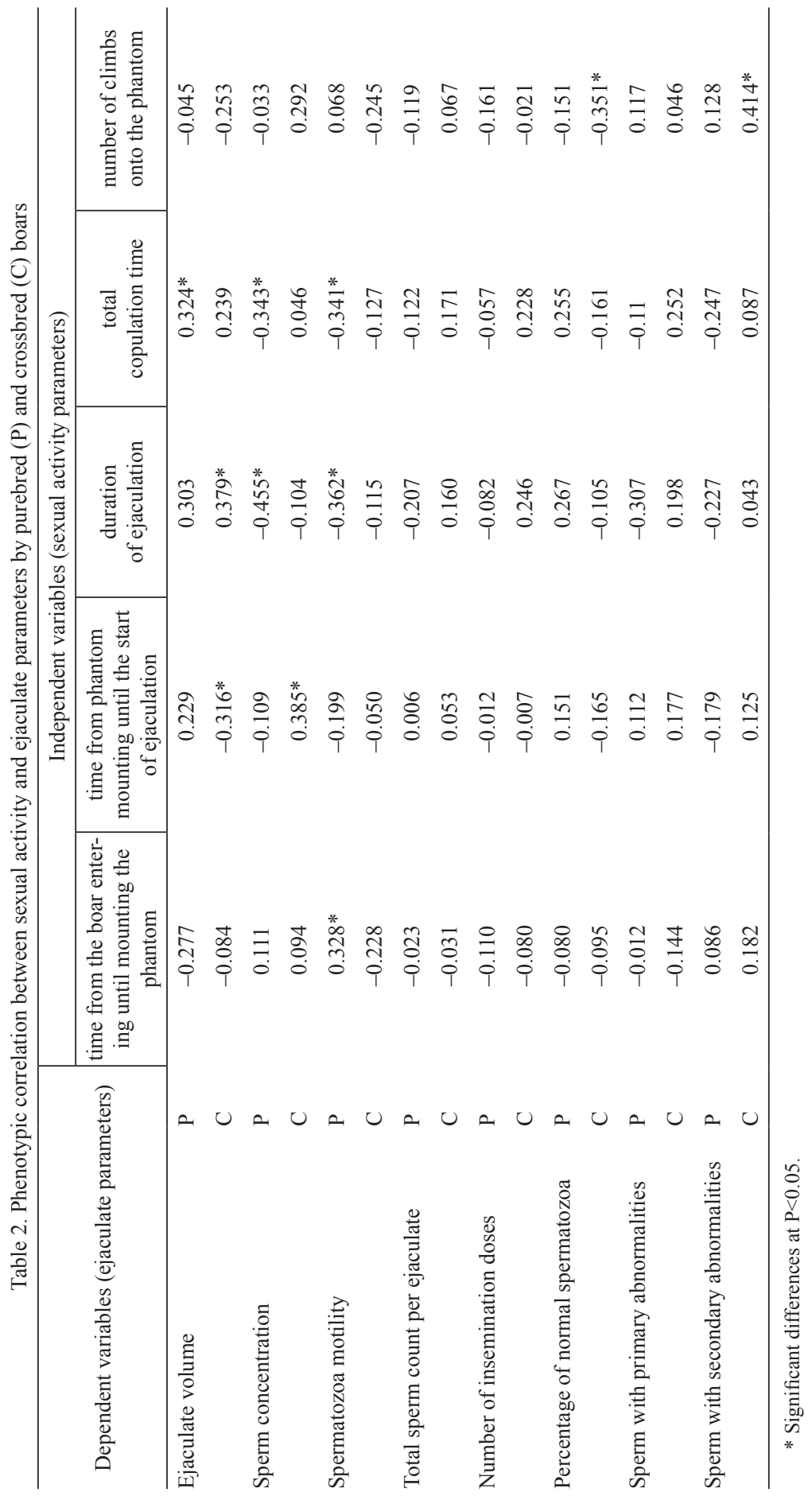


Table 2 shows data on the correlation between the ejaculate parameters and the sexual activity of the hybrids and purebred boars. The physical ejaculate characteristics only loosely depend on the time of release of particular copulatory reflexes. The data in the table suggest that physical ejaculate characteristics depend to the greatest extent on the duration of ejaculation and total copulation time. Ejaculate volume rises with prolonged ejaculation, this correlation being significant in the case of the two-breed crosses $(\mathrm{P}<0.05)$. Sperm concentration proved significantly correlated with ejaculation time and total duration of copulation, though only in the case of the purebred boars. This negative correlation was relatively high in the purebred boars ( $\mathrm{r}=-0.455$ and $\mathrm{r}=-0.343$, respectively, for the duration of ejaculation and total copulation time). It indicates an inversely proportional interrelation of ejaculate sperm concentration with the ejaculation time and total copulation time of the Duroc and Pietrain boars. The boars representing this breed were observed for an inversely proportional relationship between their sperm motility and ejaculation duration $(r=0.362)$ and total copulation time $(r=-0.341)$. The data thus indicate that a lengthening of the ejaculation time and the total copulation time in Duroc and Pietrain boars is accompanied with a fall in sperm concentration and motility and with a simultaneous increase in ejaculate volume. Ejaculate volume rises with prolonged ejaculation and total copulation period in two-breed boar hybrids. On the other hand, these libido characteristics do not affect sperm concentration and motility. The twobreed boar crosses were, in turn, observed for a stronger correlation of ejaculate volume and sperm concentration with the time between phantom mounting and ejaculation inception. As this period grows longer, the volume of two-breed hybrid ejaculates dwindles $(\mathrm{r}=-0.316)$, whereas corresponding ejaculate sperm concentration rises $(\mathrm{r}=0.385)$.

Table 3 contains detailed data presenting the level of correlation between physical ejaculate characteristics and morphological deviation incidence in spermatozoa and the analysed libido parameters of the boars, within the Duroc and Pietrain breeds and within both two-breed hybrid variants. These data confirm previous observations concerning a negligible level of correlation between ejaculate parameters and the release speed of sexual reflexes. The data show that the particular indices have different practical value for use depending on the breed or crossbreeding variant. The period between phantom mounting and ejaculation start is, as previously stated, correlated with sperm concentration in ejaculates of hybrid boars. Nevertheless, this correlation is far more pronounced in the hybrids from Pietrain sires $(r=0.560)$ than in the boar crosses by Duroc sires $(r=0.355)$. A strict correlation $(r=0.834)$ was identified between the incidence of spermatozoa with primary changes and the time between phantom mounting and ejaculation start in the Duroc boars. The data in Table 1 show Duroc boars to require the least time between mounting the phantom and starting ejaculation (63.75 seconds), whereas these boars have the highest frequency of primary (1.81\%) and secondary $(9.05 \%)$ morphological sperm deviations. It thus seems that the identified strict correlation between primary sperm alterations in this breed and the time between phantom mounting and ejaculation start is not accidental and can have practical applications. 


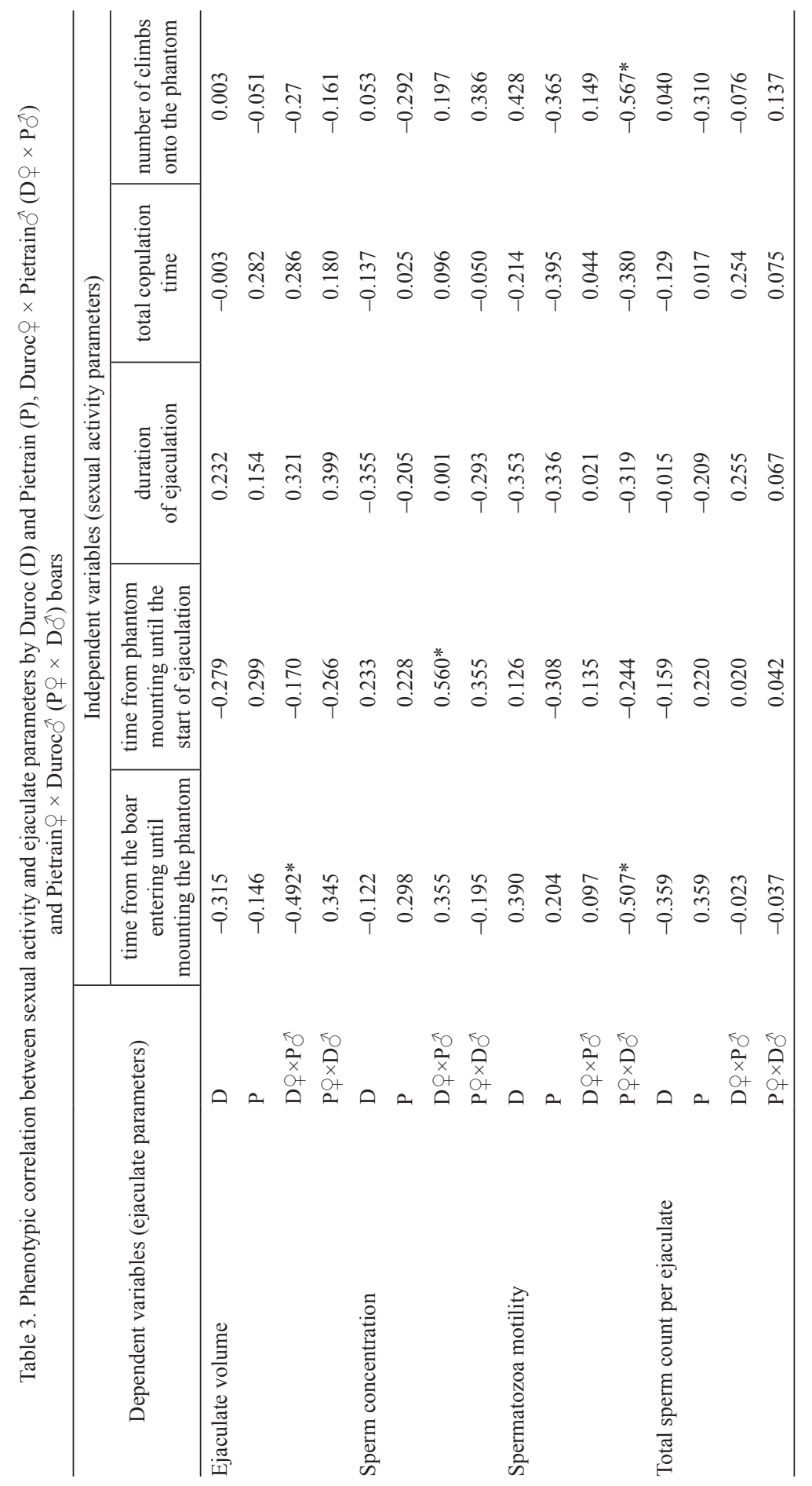




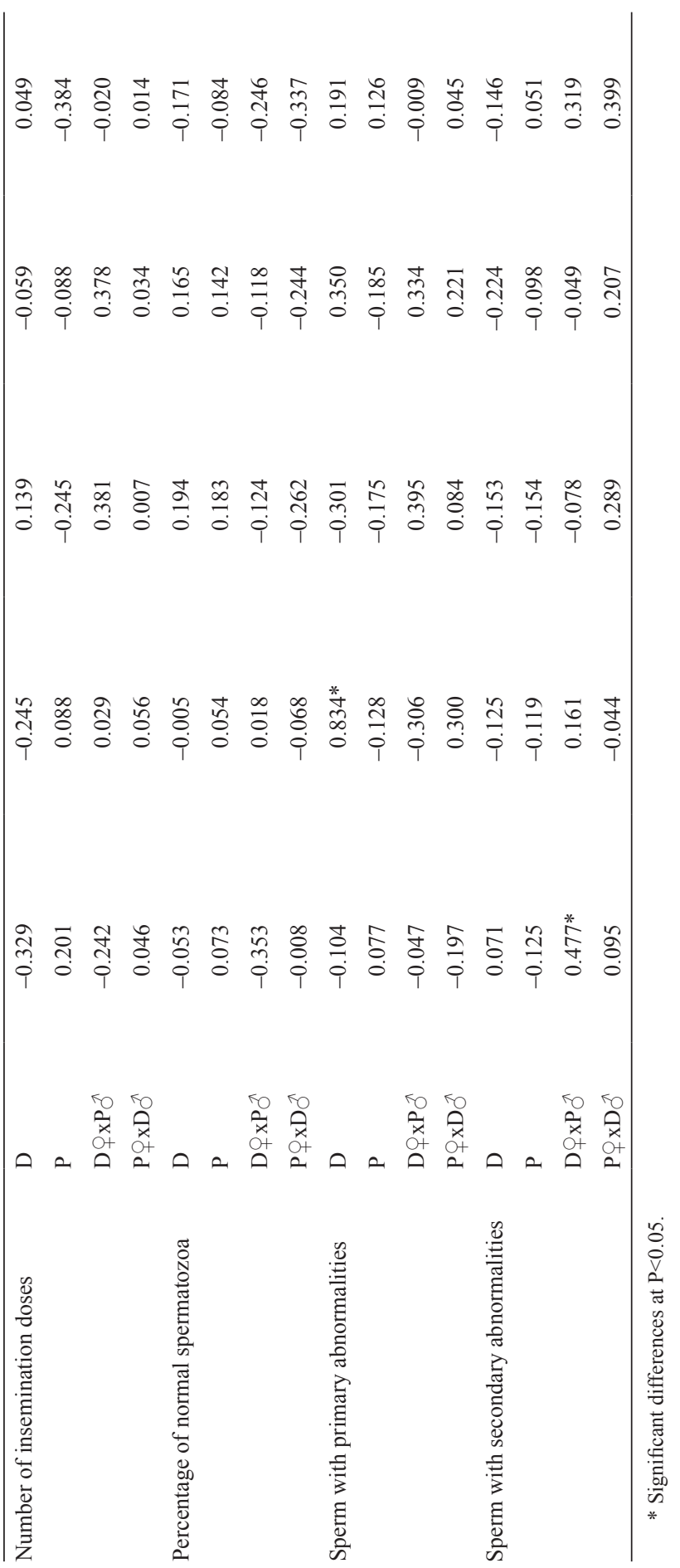




\section{Discussion}

The data presented in this paper show that sexual activity parameters are affected by the breed of the boar. The hybrid boars relatively quickly mount the phantom, as evidenced by the short periods identified between a boar's entrance into the arena and successful phantom mounting. Moreover, the hybrids by Duroc $q \times \operatorname{Pietrain}{ }^{\lambda}$, as well as purebred Duroc boars were characterized by a relatively short period between phantom mounting and ejaculation start. This, according to criteria presented by Okere et al. (2005) and Frydrychova et al. (2011), would classify these boars as males with intensive sexual interest and rapid erection and ejaculation. Despite the short time necessary for the Duroc boars to mount the phantom and start ejaculation, these males also had a short ejaculation period and a short total copulation duration, resulting in producing low-volume ejaculates. Nevertheless, sperm concentration in the ejaculates of the Duroc boars was very high. It seems a genetically perpetuated trait of this breed confirmed not only with the results of this study but also with those of numerous other ones (Smital et al., 2004; Kondracki et al., 2012). This study shows Duroc sires to have a separate character not only with regard to the release speed of sexual reflexes but also to the quantitative and qualitative ejaculate parameters. The ejaculates of the representatives of this breed were observed to have a higher incidence of spermatozoa with morphological alterations as compared with the ejaculates of the Pietrain boars and those of the two-breed crosses. This regularity was also identified in other studies (Wysokińska et al., 2006; Kondracki et al., 2012). The data presented by Banaszewska and Kondracki (2012) suggest the possibility that Duroc boars accomplish their sexual development earlier than other breeders. This is evidenced in a lower dynamics of sperm count increase as compared with other breeds, particularly a lower dynamics of age-related ejaculate volume increase and an earlier reaching of the threshold of optimal ejaculatory performance as compared with other boar breeds.

Several studies have found two-breed sires to be more sexually active than purebred boars (Czarnecki et al., 1999), as evidenced by heterosis effects that show the advantage of crossbreds over purebred sires. As in the case of other mammal species, pig males need steroid hormones to maintain an adequate level of sexual activity. A crucial role here is played by testosterone. Castrated males do not exhibit sexual interest (Williams, 2009). Sexual activity stimulation can, on the other hand, affect semen quality (Pound et al., 2002). Stimulation of males prior to ejaculate collection increases ejaculatory efficiency by facilitating sperm transport through the outlet ducts. Prostaglandins can stimulate boar testes to secrete steroid hormones (Estienne et al., 2004). Studies of older breeders have shown that prostaglandin administration can be beneficial if the breeders are required for insemination use (Estienne and Harper, 2000). On the other hand, negative effects of prostaglandin administration on ejaculate parameters have not been identified (Estienne and Harper, 2004). A natural factor that stimulates male sexual behaviour without hormonal interference is the observation of other breeders directly before entering the arena (Levis and Reicks, 2005). When approaching the semen yielding site, the boars analysed in the present 
study also had the possibility to observe the preceding male, which could act as a sexual stimulant.

The data of this study suggest that physical ejaculate characteristics mostly depend on the duration of ejaculation and total copulation time. A lengthening of the ejaculation time and of the total copulation duration in purebred Duroc and Pietrain boars is accompanied with a fall in sperm concentration and motility and with a simultaneous increase in ejaculate volume. Ejaculate volume rises with prolonged ejaculation and total copulation period in two-breed boar hybrids. Similar interrelations were identified in studies of White Duroc $\times$ Erhualian boar hybrids performed by Xing et al. (2009). Ejaculates obtained from bulls exhibiting high sexual activity have greater volumes and spermatozoa contained in them are more motile. They also contain fewer spermatozoa with morphological defects (Quririno et al., 2004). The existence of a relationship between sexual activity parameters and ejaculate characteristics and sire fertility gives rise to certain controversy. Some researchers think that such relationship does not exist. In a study of White Duroc $\times$ Erhualian boar hybrids, Ren et al. (2009) concluded that libido parameters and testosterone concentration are not correlated with ejaculate characteristics. Experiments on bulls did not identify a correlation between sexual urge parameters and semen quality, either (Galina et al., 2007). According to the authors, it is perfectly possible to obtain excellent ejaculates from bulls with low libidinal levels. Some scientists claim that hybrids may undergo spermatogenic changes due to genetic modifications caused by altered chromosome Y morphology, which may lead to changes in sperm structures (Horn et al., 2005).

Summing up, it should be stated that sexual activity parameters are affected by the breed of the boar and the crossbreeding variant. Two-breed sires are quicker to leap onto the phantom as compared with purebred boars. Hybrid Duroc $q \times$ Pietrain $\widehat{\gamma}$ boars are characterized by a short period of time between phantom mounting and ejaculation initiation. On the other hand, they yield ejaculates for a much longer time than hybrid Pietrain $q \times$ Duroc $\widehat{ }{ }^{\lambda}$ boars and purebred Duroc and Pietrain boars. Duroc boars require the least time between mounting the phantom and starting ejaculation, whereas these boars have the highest frequency of primary and secondary morphological sperm deviations. Hybrid boar ejaculates contain more spermatozoa and can provide more insemination doses than ejaculates of purebred boars. Hybrids produce high-quality semen, with an insignificant percentage of morphologically altered spermatozoa. Ejaculate parameters are chiefly correlated with ejaculation time and copulation duration. The basic ejaculate parameters (ejaculate volume, sperm concentration and sperm motility) in purebred boars chiefly depend on ejaculation time and total copulation duration, whereas in two-breed hybrids they are more affected by the length of the period between phantom mounting and the start of ejaculation.

\section{References}

B an a s zew ska D., K ondra cki S. (2012). An assessment of the breeding maturity of insemination boars based on ejaculate quality changes. Folia Biol.-Krakow, 60: 151-162. 
B 1 o m E. (1981). The morphological estimation of the spermatozoa defects of bull II. The proposal of new classification of spermatozoa defects (in Polish). Med. Weter., 37: 239-242.

$\mathrm{Ch}$ a m berla in T.S., H u g h es P.E. (1996). The influence of mating frequency and nutrition on the stimulus value of boars. Anim. Reprod. Sci., 43: 151-160.

C i e r e s z k o A., O t t o bre J.S., G l og o w s k i J. (2000). Effects of season and breed on sperm acrosin activity and semen quality of boars. Anim. Reprod. Sci., 64: 89-96.

C zarnecki R., Róży ck i M., U d ała J., Kaw ęck a M., Ka m y czek M., P ietruszka A., D e lik a t or B. (1999). The growth rate, meatiness value and reproductive performance of young Duroc boars and their hybrids with the Pietrain breed. Ann. Anim. Sci. Suppl., 3: 105-110.

E sti e n n e M.J., H a r p e r A.F. (2000). PGF $_{2 \alpha}$ facilitates the training of sexually active boars for semen collection. Theriogenology, 4: 1087-1092.

Esti e n e M.J., Harper A.F. (2004). Semen characteristics and libido in boars treated repeatedly with PGF $_{2 \alpha^{*}}$ J. Anim. Sci., 82: 1494-1498.

Estie nne M.J., Harper A.F., Knight J.W., B a r b C.R., P a m p a c ek G.B. (2004). Sexual behavior after treatment with prostaglandin- $\mathrm{F}_{2 \alpha}$ in boars with suppressed concentrantions of gonadal steroids. App. Anim. Behav. Sci., 89: 53-57.

Frydrychova S., Opletal L., Macáková K., Lustyková A., Rozkot M., Lipens ký J. (2011). Effects of herbal preparation on libido and semen quality in boars. Reprod. Dom. Anim., 46: 573-578.

G a li in a C.S., H or n M.M., M o li in a R. (2007). Reproductive behaviour in bulls raised under tropical and subtropical conditions. Horm. Behav., 52: 26-31.

Hemsworth P.H., Tilbrook A.J. (2007). Sexual behavior of male pigs. Horm. Behav., 52: $39-44$.

Horn M.M., M or a e s J.C.F., J a u m e C.M., E d e 1 w e i s s M.I.A., R o s a d o A. (2005). Reproductive deficiency in bulls from synthetic breeds according to the type of crossbreed and the morphology of the Y chromosome. Genet. Mol. Biol., 28: 225-229.

K a w ęck a M., P ietruszka A., Jac yno E., Czarnecki R., Ka m y czek M. (2008). Quality of semen of young boars of the breeds Pietrain and Duroc and their reciprocal crosses. Arch. Tierz., 51: $42-54$.

Kn e ch t D., Ś rodoń S., S zu l c K., Duzińsk i K. (2013). The effect of photoperiod on selected parameters of boar semen. Livest. Sci., 157: 364-371.

Kondracki S., I w an in a M., Wy sokińska A., Huszno M. (2012). Comparative analysis of Duroc and Pietrain boar sperm morphology. Acta Vet. Brno, 81: 195-199.

Le vis D.G., F ord J.J., Christen se n R.K. (1997). An evaluation of three methods for assessing sexual behaviours in boars. J. Anim. Sci., 75: 348-355.

L e v is D.G., R e i c k s D.L. (2005). Assessment of sexual behavior and effect of semen collection pen design and sexual stimulation of boars on behavior and sperm output - a review. Theriogenology, 63: 630-642.

Okere Ch., Joseph A., Ezekwe M. (2005). Seasonal and genotype variations in libido, semen production and quality in artificial insemination boars. J. Anim. Vet. Adv., 4: 885-888.

P e th e r i c k J.C. (2005). A review of some factors affecting the expression of libido in beef cattle, and individual bull and herd fertility. Appl. Anim. Behav. Sci., 90: 185-205.

Pound N., Javed M.H., Ruberto C., Anis Shaikh M., Del Valle A.P. (2002). Duration of sexual arousal predicts semen parameters for masturbatory ejaculates. Physiol. Behav., 76: $685-689$.

Quririno C.R., Bergmann J.A.G., Vale Filho V.R., Andrade V.J., Reis S.R., Mend on c a R.M., F o n s e c a C.G. (2004). Genetic parameters of libido in Brazilian Nellore bulls. Theriogenology, 62: 1-7.

Ren D., Xing Y., Lin M., Wu Y., Li K., Li W., Yang S., Guo T., Ren J., Ma J., Lan L., $\mathrm{H} \mathrm{u}$ a $\mathrm{g}$ L. (2009). Evaluations of boar gonad development, spermatogenesis with regard to semen characteristics, libido and serum testosterone levels based on Large White Duroc $\times$ Chinese Erhualian crossbred boars. Reprod. Dom. Anim., 44: 913-919.

S mital J., De S o u s a L.L., Mohns en A. (2004). Differences among breeds and manifestation of heterosis in AI boar sperm output. Anim. Reprod. Sci., 80: 121-130.

S o n d e r m a n J.P., L u e b b e J.J. (2008). Semen production and fertility issues related to differences in genetic lines of boars. Theriogenology, 70: 1380-1383. 
Willi a m s S. (2009). Assessment of the boar reproductive efficiency: Physiology and implications. Rev. Bras. Reprod. Anim. Belo Horizonte, 6: 194-198.

Wolf J., S mital J. (2009). Quantification of factors affecting semen traits in artificial insemination boars from animal model analyses. J. Anim. Sci., 87: 1620-1627.

Wy s o k ińs k a A., K on d ra ck i S., B a n a s z e w s k a D. (2006). Application of spermiogram classification in the evaluation of the semen morphology of Duroc $\times$ Pietrain crossbreds and purebred Duroc and Pietrain. Anim. Sci. Pap. Rep., 24: 319-325.

Wysokińska A., Kondracki S., Kowalewski D., Adamiak A., Muczyńska E. (2009). Effect of seasonal factors on the ejaculate properties of crossbred Duroc $\times$ Pietrain and Pietrain $\times$ Duroc boars as well as purebred Duroc and Pietrain boars. Bull. Vet. Inst. Pulawy, 53: $677-685$.

Xing Y., Ren J., Re n D., Gu o Y., Wu Y., Yang G., Ma o H., B renig B., H u ang L. (2009). A whole genome scanning for quantitative trait loci on traits related to sperm quality and ejaculation in pigs. Anim. Reprod. Sci., 114: 210-218.

Received: 14 XI 2013

Accepted: 4 III 2014 